\section{Ueber Infusorien im Magen und im Darmkanal des Menschen und ihre klinische Bedeutung.)}

Von Dr. Paul Cohnheim in Berlin.

M. H.! Die Protozoën oder Urthiere, diese einzelligen Organismen, welche in der Rangordnung unserer Fauna am tiefsten stehen, haben gegenwärtig bekanntlich eine grosse Bedeutung für die menschliche Pathologie gewonnen, weil man in ihnen die Erreger eines Theiles der Infektionskrankheiten gefunden hat oder $\mathrm{zu}$ sehen geneigt ist.

Die beim Menschen bis jetzt beobachteten Protozoën zerfallen, wenn ich der Eintheilung Braun's (1) in seinem bekannten Werke über die thierischen Parasiten des Menschen folgen darf, in drei grosse Klassen, die Rhizopoden, zu denen die bekannten und in der Dysenteriefrage bedeutsamen Amoeben gehören, die Sporozoën, welche beispielsweise die Malariaplasmodien umfassen und welchen auch die bisher bei den malignen Geschwülsten aufgefundenen Parasiten zugerechnet werden, und schliesslich die Infusorien.

Ueber das Vorkommen und die Bedeutung dieser im gesammten Verdauungstraktus des Menschen möchte ich Ihnen im Folgenden berichten.

M. H.! Ich hatte Gelegenheit, eine grössere Anzahl einschlägiger Fälle unter dem Krankenmaterial meines Ambulatoriums für Magen - und Darmkrankheiten zu beobachten, und da ich bezüglich der klinischen Bedeutung der Infusorien zu Schlüssen gelangt bin, welche von der bisherigen Meinung abweichen und mir für die Diagnostik der Intestinalerkrankungen von Bedeutung zu sein scheinen, so hielt ich mich für berechtigt, in dieser Frage hier das Wort zu ergreifen.

Ich will vorweg bemerken, dass ich in meinen Fällen ausschliesslich Geisselinfus orien (Flagellaten seu Mastigophora) gefunden habe, während mir ein Fall mit Wimperinfusorien (Ciliata), zu denen beispielsweise das bekannte bei Schweinezïchtern besonders häufig vorkommende Balantidium coli gehört, leider nicht zur Beobachtung kam.

Ich habe drei Species von Geisselinfusorien bei meinen Fällen gesehen: Darm.

1. Trichomonas hominis im Munde, Oesophagus, Magen,

2. Megastoma entericum, im Magen und Darm.

3. Plagiomonas hominis, im Darm.

Von den Trichomonaden sah ich verschiedene Unterarten, worauf ich weiter unten zurückkommen werde, ausserdem fanden sich in allen Fällen gleichzeitig mit den Infusorien auch zahlreicheAmoeben in allen Phasen der Entwickelung, lebend, todt und encystirt, ferner in einem Falle (siehe unten) ein Radiolarion in den Dejektionen.

M. H.! Die Morphologie und Biologie dieser Infusorien sind seit langem bekannt; ich begnüge mich daher, Ihnen diese Zeichnungen herumzugeben, welche theils nach der Natur, theils nach den bekannten Lehrbüchern von Braun (1) und Doflein (2) angefertigt sind.

Sie sehen die elliptischen, etwa $8-12 \mu$ grossen Trichomonad en, mit einer Schwanzgeissel und zwei bis drei Kopfgeisseln, ferner die exquisit birnförmigen Megas to m en (seu Cercomonaden) mit acht Geisseln und die Plagiomonaden mit lang ausgezogenem Schwanzende, zwei Kopfgeisseln und einer Art Mundöffnung. Dies letztere Infusorium wurde beim Menschen bis dato nư im Urin aufgefunden. Ferner sehen Sie neben lebenden auch todte und encystirte Formen der Infusorien gezeichnet.

Wenn ich nun des Näheren auf mein Beobachtungsmaterial, zunächst soweit es den Magen angeht, eingehen darf, so betrifft dies einen Fall von Oesophagus- und fünf Fälle von Magenkrebs. Die Fälle von Magencarcinom waren ausschliesslich solche, welche ihren Sitz nicht am oder nahe dem Pylorus, sondern mehr der Cardia zu hatten, die also zu keinerlei Stenosen- und Retentionserscheinungen geführt haben konnten.

Ich will hier gleich hinzufügen, dass auch die übrigen in der Litteratur mitgetheilten Fälle von Infusorienbefund im Magen nichtpylorische Magenkrebse betrafen.

Ein ungeübter Untersucher, meine Herren, könnte wohl die

1) Vortrag, gehalten im Verein für innere Medizin am 12. Mai 1902. gewöhnlichen Süsswasserinfusorien, welche sich mitunter auch im stagnirenden, zur Verdünnung der Präparate benutzten Laboratoriumswasser entwickeln, mit den uns interessirenden Flagellaten verwechseln. In meinen Fällen war eine derartige Täuschung ausgeschlossen. Das verdächtige, schleimig-eitrige, meist sanguinolente Substrat wurde mit sauberer Schlundsonde entnommen, in ein trockenes, erwärmtes Petri'sches Schälchen gebracht und sofort unverdünnt mikroskopirt, eventuell zur späteren Untersuchung in den Thermostaten gestellt. Um Ihnen die Unterschiede zwischen gewöhnlichen Süsswasserinfusorien und unseren Flagellaten ad oculos zu demonstriren, habe ich mir erlaubt, dort zwei Präparate aufzustellen; das eine enthält G ei s selthierchen, die sich im Glasgefäss einer Hyazinthe ${ }^{1}$ ) entwickelt haben, das andere lebende Trichomonaden, die vor einer halben Stunde einem meiner Patienten entnommen wurden.

Die von mir beobachteten Fälle sind folgende:

\section{Oesophagus.}

Fall 1. 54jähriger Grünkramhändler, aufgenommen am 7. September 1901. 'Das Leiden begann sechs Wochen vor Aufnahme des Patienten in die Poliklinik mit Drücken und Wühlen im Epigastrium nach der Nahrungsaufnahme, besonders nach harten Speisen; Appetit schleeht, Stuhl angehalten; leichte Schluckbeschwerden, in letzter Zeit Erbrechen von Schleim und Speisen; erhebliche Abmagerung.
F1g. 1. $12-15 \mu$

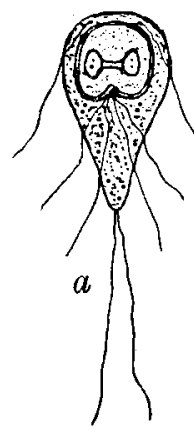

Megastoma entericum. $a$ vorn, $b$ seitlich, $c$ todt,
$d$ encystirt.
Fig. 2.

$10 \mu$.

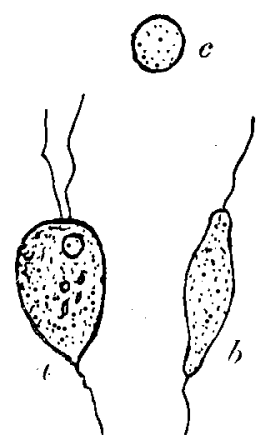

Trichomonas hominis.

$a$ intestinalis, $b$ elongatum, $c$ encystirt.

Magerer, anämischer Patient mit leidendem Gesichtsansdruck; Herz und Lunge ohne besonderen Befund, ebenso Abdomen. Weiche, mittelstarke Sonde stösst in $35 \mathrm{~cm}$ von der Zahnreihe auf ein Hinderniss. - Am 28. September wird der Oesophagus ausgespült, hinterher eine Oeleingiessung gemacht, um die Schluckbeschwerden zu lindern.

Im Spülwasser mikroskopisch: Amoeben, todte und lebende, rothe und weisse Blutkörperehen und einzelne lebende Infusorien, sehr beweglich, zur Gruppe der Trichomonaden gehörig.

1. Oktober 1901. Schluckbeschwerden bei täglicher Oelung besser linker Sternalrand im vierten Interkostalraum sehr druckempfindlich. Patient Starb am 19. März 1902 an Entkräftung.

1) Zum Studium der klinischen Protozoënfrage seien Medizinern die Werke von Braun (1), Doflein (2), Mosler und Peiper (3) und Pfeifer (4) empfohlen; zum Studium der Infusorien überhaupt, besonders der Wimperinfusorien, über welche eine ungeheure Litteratur vorliegt, eignen sich die folgenden Werke und Schriften: Stein, Der Organismus der Infusionsthiere. Leipzig 1867, Bd. II. - Zürn, Die Schmarotzer auf und in dem Körper unserer Haussäugethiere. Weimar 1874, Bd. II, S. 441 und II. Auflage 1887, S. 790. - Schuberg, Die Protozoën des Wiederkäuermagens I. Zoologische Jahrbücher, 1888 , Bd. III. - Richard Eberlein, Ueber die im Wiederkäuermagen vorkommenden ciliaten Infusorien. Zeitschrift für wissenschaftliche Zoologie 1895, Bd. LIX, S. 233. - A dolf Günther, Weitere Beiträge zur Kenntniss des feineren Baues einiger Infusorien aus dem Wieder känermagen und dem Coecum des Pferdes. Ibidem 1899, Bd. LXIV, 1900, Bd. LXVII. - Beim praktischen Studium der Infusionsthierchen beginne man mit der mikroskopischen Untersuchung von Tümpelwasser, Heuinfusen, Wurzelwasser von Zimmergewächsen und dem Inhalt der zwei ersten Abtheilungen des Wiederkäuermagens vom frischgeschlachteten Rind, des Pferdecoecums und Schweinedarms; für die Wimperinfusorien, die $1 / 20-1 \mathrm{~mm}$ gross werden, genügt $\mathrm{sch}$ wache Vergrösserung, für die Flagellaten, die kaum über $15-20 \mu$ messen, ist starke nöthig. 
Eine ösophagoskopische Untersuchung wurde nicht vorgenommen; trotzdem war an der Diagnose "Speiseröhrenkrebs" nicht zu zweifeln: zjemlich plötzlich entstandenes Schluckhinderniss, Kachexie, Schmerzstelle am Sternum etc.

Der Infusorienbefund sprach für Ulzeration des Neoplasmas.

\section{Magen.}

Fall 2. 44jährige Hausdienersfrau E. B., aufgenommen am 21. Dezember 1899. Anamnese ohne Besonderheiten; sehr kachektische Frau, in der linken Partie des Epigastriums ein querverlaufender höckeriger, harter, etwa birnengrosser, wenig druckempfindlicher Tumor.

22. Dezember. Nüchtern eitriger Schleim (keine Speisereste), darin neben rothen und weissen Blutzellen zahllose Amoeben und Trichomonaden. Totale Achylie.

Patientin ist kurze Zeit darauf gestorben.

Die Diagnose wurde hier durch den Infusorienbefund nur bestätigt.

Fall 3. 64jähriger Arbeiter C. R., aufgenommen am 31. Januar 1902. Sèit sechs Wochen Magenbeschwerden, besonders nach harten Speisen, öfters Erbrechen und Zusammenlaufen von Wasser im Munde, Appetitlosigkeit, starke Abmagerung, Verstopfung.

Abgemagerter, blasser Patient, Foetor ex ore, Herz und Lungen ohne Besonderheiten, Haut trocken und schilferig, einige kleine Angiome, Leber etwas vergrössert palpabel, Colon transversum contrahirt fühlbar, Hernia inguinalis dextra, im Abdomen, insbesondere imEpigastrium kein Tumor zu tasten.

Im ausgeheberten, speisefreien, alkalisch reagirenden, schleimigeitrigen und etwas san guinolenten Mageninhalt findet man in jedem Präparat vereinzelte, sehr lebhafte Geissel. und Fortbewegung zei- $a$ lebend, $b$ encystirt. gende Infusorien ron

elliptisch-kugeliger Gestalt, etwa $8-9 \mu$ gross, die als Trichomonaden rekognoszirt werden.

Die Diagnose wird auf diesen Befund hin, trotzdem Kachexie und Tumor fehlen, auf ein ulzerirendes nichtpylorisches Magencarcinom gestellt. - Der Patient kam nicht in die Poliklinik zurück, in die er nur zur Diagnosenstellung geschickt war. Nach Bericht des behandelnden Arztes bekam der Kranke zwei Wochen später eine sehr schwere Magenblutung und liegt jetzt (vier Monate nach der Erhebung des Infusorienbefundes) hoffnungslos darnieder. Ein Tumor ist auch jetzt noch nicht fühlbar.

Fall 4. 69jähriger Tischler C. Sch., aufgenommen am 16. Februar 1901. Seit $1 / 2 \mathrm{Jahr}$ Magenbeschwerden, Uebelkeit und Wehgefühl nach dem Essen, viel Aufstossen, schmerzhaftes Hungergefühl, besonders Nachts, durch geringe Nahrungsaufnahme unterdrückt, kein Erbrechen.

Leidlich genährter, anämischer Patient, negativer palpatorischer Magenbefund, $\mathrm{k}$ ein Tumor tastbar.

18. Februar. Magen nüchtern nach Probeabendbrot (ein Teller Rosinenmehlsuppe und ein belegtes Brödchen) vollkommen speisefrei (also Motilität gut); die Sonde bringt etwas sanguinolenten Schleim heraus, in dem sich neben Amoeben vereinzelte lebende Trichomonaden vorfinden. Probefrühstück total achylisch; G. A. gleich 6, keine freie oder gebundene $\mathrm{HCl}$; Pepsin und Lab in Spuren.

In den folgenden Wochen keine Besserung; Probefrühstück stets achylisch, G. A. stets 6 , stets mit blutigem Schleim vermengt, Gewicht am 9. März 151 Pfund, am 4. April 137 Pfund. Tumor immer noch nicht palpabel

Patient starb nach mündlichem Bericht der Gattin am 14. April 1902, also etwa ein Jahr später, nachdem er in der Zwischenzeit zweimal auf einer stationären Klinik verpflegt worden war. In den letzten Monaten der Krankheit waren Schluckbeschwerden aufgetreten. Die Krankenhausdiagnose lautete: Magen- und Speiseröhrenkrebs.

Fall 5. 62 jähriger Sattler R. V., aufgenommen am 8. Januar 1902. Patient leidet seit 16 Jahren an Muskelreissen, seit etwa einem Jahre an Magenbeschwerden, bis dahin magengesund, starke Appetitlosigkeit; Magendrücken beständig, auch Nachts; Völlegefühl nach dem Essen; keine Schluckbeschwerden, starke Abmagerung, Stuhl regelmässig, ein. mal täglich, fest, geformt; Lues aut Potus negatur; von Juli bis Dezember 1901 besschwerdefrei, dann Rückfall, Ende Dezember 1901, also acht bis zehn.Tage vor der Aufnahme in die Poliklinik, Erbrechen, sonst nie Erbrechen.

Sehr magerer, anämischer Patient, von kachektischem Aussehen, kräftigem Körperbau, gebückter Haltung, kein Habitus enteroptoticus; breiter Thorax, stumpfer Angulus costarum; am Rücken zehn, an der Brust drei kleine Angiome; ${ }^{1}$ ) Herz und Lungen ohne Besonderheiten, Abdomen kahnförmig eingesunken, in der Mitte zwischen Nabel und Processus xiphoideus eine druckempfindliche Stelle mit undeutlich fühlbarer rundlicher Resistenz, Gewicht $61 \mathrm{~kg}$ (früher $75 \mathrm{~kg}$ ) in Kleidern.

9. Januar. Im nüchternen Magen, 14 Stunden nach der letzten Nahrungsaufnahme $8-10 \mathrm{ccm}$ hellgrünliche, schleimig-eitrige Flüssigkeit, keine Nahrungsrückstände; bei mikroskopischer Untersuchung findet man in der Flüssigkeit grosse Mengen lebender und schon abgestorbener Infusorien, welche leicht als zur Spezies Megastoma entericum gehörig erkannt werden; die Reaktion der Flüssigkeit ist stark alkalisch; die Megastomen liegen theils einzeln, theils in Gruppen von 10-15 Exemplaren zusammen; ihre Bewegung ist eine äusserst lebhafte, der ganze Körper schnellt oft zusammen, und Schwanz- und Mittelgeisseln bewegen sich besonders lebhaft; encystirte Formen dieser Infusorien (s. Fig. 1) werden im Mageninhalt nicht gefunden; an denjenigen Individuen, welche in Folge der Abkühlung, der Belichtung und der sonstigen ausserhalb des Wirthes schädlich einwirkenden Einflüsse bereits die lebhafte Beweglichkeit eingebüsst haben und nur noch ruhig auf der Seite oder auf dem Rücken liegend ihre Geisseln mehr oder weniger schnell wie ein Wimpel im Winde bewegen, sieht man bei Oelimmersion alle Details des Baues, die Delle, den vorspringenden Stachel, die zwei Kerne und die acht Geisseln; letztere besonders schön an Präparaten, die einige Sekunden über die Dämpfe einer $1 \%$ igen Osmiumsäurelösung gehalten wurden. Conservirung der Thierchen in Glycerin misslang.

Ausser den Infusorien sieht man grosse, vakuolenhaltige, amoebenähnlicheZellen, aber ohne amoeboide Bewegungen, daneben viele weisse und rothe Blutkörperchen und Bakterien.

Im Probefrühstück fehlen Salzsäure, Lab und Pepsin. Patient bekommt eine flüssig-breiige, sehr butterreiche Diät und Condurangoextrakt.

14. Januar. Nüchtern wieder grosse. Mengen von lebenden $\mathrm{Me}$ gastomen, Status idem.
$20 \mu$.

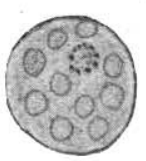

c
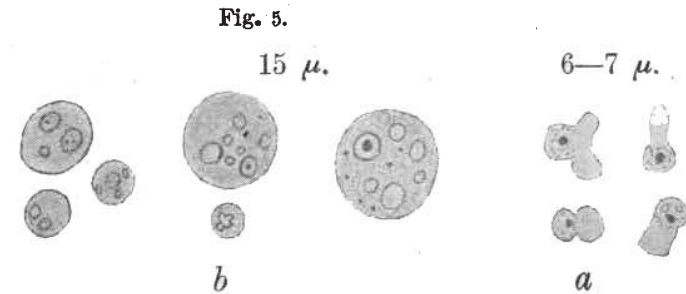

Darmamöben bei einem Fall von schwerer Gastro-Enteritis chronica.

$a$ lebend, $b$ todt, $c$ encystirt.

15. Januar. In dem gestrigen Mageninhalt, der im Thermostaten gestanden, heute nichts mehr von Infusorien erkennbar.

22. Januar. Befinden etwas besser; nüchtern wieder in den grünlichen Flocken zahllose lebende und todte, keine encystirten Megastomen, einzeln oder haufenweise bis 30 Stüick; sie werden noch nach zweistïndigem Verweilen im Thermostaten lebend angetroffen, nach vier Stunden nur noch todte Exemplare auffindbar; viele Vakuolenzellen, rothe und weisse Blutzellen.

29. Januar. Derselbe Befund im nüchternen Magen.

1. Februar. Abends $7 \mathrm{Uhr}, 7 \frac{1}{2}$ Stunden nach der letzten Nahrungsaufnahme, keine Infusorien auffindbar.

3. Februar. Nüchtern wieder viele Infusorien, $3 / \mathrm{kg}$ Zunahme.

10. Februar. $1^{1 / 4} \mathrm{~kg}+$, Appetit besser; nüchtern viele Infusorien.

13. Februar. Derselbe Befund.

17. Februar. Patient wird Herrn Prof. Eberle in ${ }^{2}$ ) von der Thierärztlichen Hochschule vorgestellt und $\mathrm{ihm}$ in dessen Laboratorium der Inhalt des nüchternen Magens entnommen. Herr Prof. Eberlein hatte die Gïte, die Präparate zu besichtigen und die Identität der bei meinem Patienten aufgefundenen Infusorien mit dem Megastoma entericum zu bestätigen. Ich will nicht verfehlen, ihm für seine grosse Liebenswürdigkeit an dieser Stelle meinen Dank auszusprechen.

20. Februar. Im Stuhl des Patienten ungeheure Mengen von $\mathrm{Me}$ gastomencysten; Gewichtszunahme $2 \mathrm{~kg}$, heute nur noch wenige schwach lebende oder todte Exemplare auffindbar. Der Patient wird Herrn Geheimrath v. Leyden vorgestellt, der sich von der Richtigkeit des Befundes iuberzeugte. Ich spreche ihm an dieser Stelle meinen wärmsten Dank für seine liebenswürdige Bereitwilligkeit, die Präparate durchzusehen, aus. findbar.

10. März. $3 \mathrm{~kg}$ Zunahme; im Mageninhalt keine Infusorien auf-

1) Auftreten von Hautangiomen soll nach Leser ein Zeichen von Carcinose innerer Organe sein, weshalb wir darauf achteten. Münchener medizinische Wochenschrift 1901, No. 51.

2) Siehe Anmerkung S. 206. 
14. März. Abends, sieben Stunden nach dem Mittagessen, nur zwei bis drei todte Infusorien im Mageninhalt zu finden.

20. März. Wieder reichlich lebende Megastomen im grünlichen, schleimig - eitrigen, alkalischen Inhalt des nüchternen Magens; ausserdem viel Vakuolenzellen und Amoebencysten (siehe Fig. 5 u. 6).

9.Mai. Patient musste wegen rheumatischer Schmerzen in den Beinen zu Hause bleiben. Gewicht $63,5 \mathrm{~kg}$, Magen-

Fig. 6.

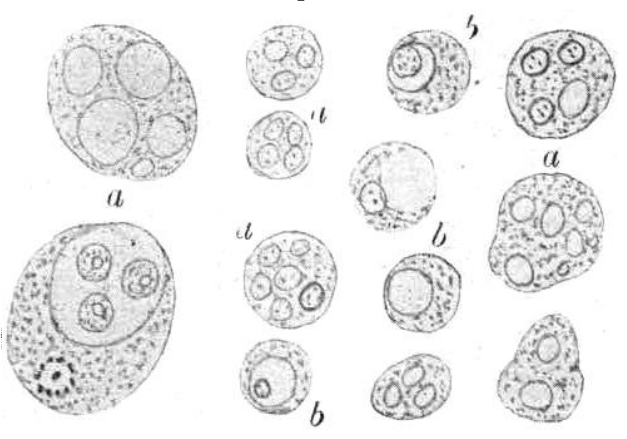

Vakuolenzellen bei Carcinom und Gastritis atrophicans.

$a$ Geschwulstzellen, $b$ Epithelzellen, hyalin degenerirt.

drücken; nüchtern im

sanguinolenten Schleim keine Megastomen, dagegen Trichomonaden.

23. Mai. 65,5 kg, Oedeme nicht stärker, Fettpolster im Abdomen verstärkt, keine Resistenz mehr im Epigastrium auffindbar; Aussehen blass, leicht kachektisch; weiche Sonde stösst in $55 \mathrm{~cm}$ auf unüberwindlichen Widerstand; nüchtern wieder nur Blut, Eiter, Schleim und Trichomonaden, keine Megastomen. Im Zahnbelag der kariösen Zähne des Kranken werden dieselben Trichomonaden aufgefunden, neben vielen Spirillen.

4. Juni. Befinden des Patienten bei Suppen- und Puréediät leidlich, Stuhl regelmässig, $64,5 \mathrm{~kg}$; leichte Knöchelödeme; kein Tumor im Epigastrium; nüchtern wieder eitriger Schleim mit vielen lebenden Megastomen, die $1 \frac{1}{2}$ Stunden nach ihrer Herausnahme noch lebend sind. Keine Trichomonaden.

12. Juni. Hente wieder enorme Mengen lebender Megastomen, die etwa drei Stunden lang bei Tagestemperatur (warmer Sommertag) beweglich beobachtet werden. Keine Trichomonaden. Befinden dasselbe. Gewicht $65 \mathrm{~kg}$. (Vorstellung bei v. Leyden.) ${ }^{1)}$

Fall 6. 54jähriger Maurer F. B., aufgenommen am 7. Mai 1902. 1886 vier Monate lang an Magenkatarrh behandelt; seit etwa sechs Wochen Appetitlosigkeit, Magendrücken, Völlegefühl, Verschlimmerung nach dem Essen, Verstopfung; kein Erbrechen, nur leeres Aufstossen, erhebliche Abmagerung; keine Schluckbeschwerden, grosse Mattigkeit.

Magerer, blasser Patient. Herz und Lungen ohne Besonderheiten. Leber sehr vergrössert, reicht bis zur Nabelhorizontalen, sehr hart, hat knollige Tumoren in beiden Lappen, sehr druckempfindlich; kein Ascites.

9. Mai. Nach Probeabendéssen keine Speisereste; nur $6-7 \mathrm{ccm}$ blutig-eitrigen Schleims, leicht fötide riechend, ohne Infusorien. Probefrühstück total achylisch, G. A. 8. Verórdnung: Condurango, Suppen- und Breidiät. Lab in Spuren $1 / 20+K, 1 / 40+F l$

10. Mai. Leichter Widerstand an der Cardia bei Einführung einer weichen Sonde; nüchtern leicht fötider, sanguinolenter, schleimigeitriger Inhalt mit zahlreichen, in sehr lebhafter schnellender Bewegung befindlichen Trichomonaden von zwei verschiedenen Spezies, Trichomonas elongata und elliptica. Noch drei Stunden nach Herausnahme lebend, viel Spirillen. Infusorien meist in der Nähe der Plattenepithelien und Leptothrixfäden.

11. Mai. Im gestrigen Substrat noch einzelne lebende Trichomonaden.

12. Mai. Im Zahnbelag lebende Trichomonaden.

Patient ist weiter in Beobachtung.

(Fortsetzung folgt.) 